\title{
SLIP-DECELERATION CONTROL IN ANTI-LOCK BRAKING SYSTEMS
}

\author{
Sergio M. Savaresi ${ }^{\dagger}$, Mara Tanelli ${ }^{\dagger}$, Carlo Cantoni ${ }^{*}$, Demos Charalambakis ${ }^{*}$, \\ Fabio Previdi ${ }^{\S}$, Sergio Bittanti ${ }^{\dagger}$ \\ ${ }^{\dagger}$ Dipartimento di Elettronica e Informazione, Politecnico di Milano, Piazza L. \\ da Vinci, 32, 20133 Milano, ITALY. \\ *BREMBO S.p.A., Via Brembo, 25, 24035, Curno (BG), ITALY. \\ ${ }^{\S}$ Dipartimento di Ingegneria Gestionale e dell'Informazione, Università di \\ Bergamo, Via Marconi 5, 24044 Dalmine (BG), ITALY
}

\begin{abstract}
In road vehicles, wheel locking can be prevented by means of closedloop Anti-lock Braking Systems (ABS). Two output measured variables are usually considered for regulation: wheel- deceleration and wheel longitudinal slip. The traditional controlled variable used in ABS is the wheel deceleration, since it can be easily measured with a simple wheel encoder; however, it can be dynamically critical if the road-surface rapidly changes. On the other hand, the regulation of the longitudinal slip is much robust from the dynamical point of view, but the slip measurement is critical, since it requires the estimation of the longitudinal vehicle speed. In this work a control strategy is proposed, where the regulated variable is a combination of wheel deceleration and longitudinal slip. Copyright (C) 2005 IFAC
\end{abstract}

Keywords. Automotive systems; Anti-lock Braking Systems; vehicles control.

\section{INTRODUCTION}

Most of the modern road vehicles are equipped with electronic Anti-lock Braking Systems (ABS). ABS can greatly improve the safety of a vehicle in extreme circumstances, since it can maximize the longitudinal tire-road friction, while preventing lateral forces from going to zero (Drakunov et al., 1995, Gissinger et al., 2003, Johansen et al., 2003, Layne et al., 1993, Solyom et al., 2004, Wellstead and Petit, 1997). ABS is a closed-loop control system, where two output measured variables are used: the wheel deceleration and the wheel slip.

The traditional controlled variable used in ABS is the wheel deceleration, since it can be easily measured; however, a regulation loop closed on the wheel deceleration may be critical if the road-surface rapidly changes. Henceforth, deceleration-based control strategies require the on-line estimation of road-tire friction characteristics (Gustafsson, 1997, Ono et al., 2003). The regulation of the wheel longitudinal slip is simpler from the dynamical point of view, and the slip set-point does not require online adaptation. However, the main drawback of slipcontrol is that the accurate measurement of the longitudinal slip is critical.

The current trend is to move from decelerationcontrol to slip-control, which is very attractive since it can be straightforwardly extended from ABS applications to more sophisticated Electronic Stability Control (ESC) systems (Kiencke and Nielsen, 2000). The challenge for slip-control is, henceforth, to alleviate its sensitivity to poor slip measurements. In this work a new control strategy is proposed. The basic idea of this control approach is very simple: the regulated variable is a convex combination of the wheel slip and deceleration. This 
strategy turns out to be very powerful and flexible: it can reduce the obnoxious effects of poor slip measurements, while avoiding the poor dynamical behavior of deceleration control. This control approach is named "Mixed Slip-Deceleration" (MSD).

\section{SYSTEM DESCRIPTION}

For the design and testing of braking control algorithms, a simple but effective quarter-car model is typically used. The model is given by the following equations (Fig.1):

$\left\{\begin{array}{l}J \dot{\omega}=r F_{x}-T_{b} \\ m \dot{v}=-F_{x}\end{array}\right.$

In (1), $\omega$ is the angular speed of the wheel; $v$ is the longitudinal speed of the vehicle; $T_{b}$ is the braking torque; $F_{X}$ is the longitudinal road-tire contact force; $J, m$ and $r$ are the momentum of inertia of the wheel, the quarter-car mass, and the wheel radius, respectively.

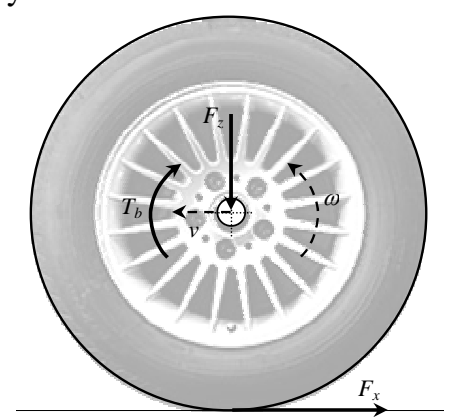

Fig.1. Quarter-car model.

Throughout the paper, the normalized linear wheel deceleration $\eta=-\dot{\omega} r / g$ will be used. Observe that $\eta$ is the linear deceleration (expressed in $\left[\mathrm{m} / \mathrm{s}^{2}\right]$ ) of the contact point of the tire, normalized with respect to the gravitational acceleration $g$. It is particularly useful since it can be easily compared with the vehicle deceleration. The dynamic behavior of the system is hidden in the expression of $F_{x}$, which depends on the state variables $v$ and $\omega$. The most general expression of $F_{x}$ is quite complicated, since $F_{x}$ depends on a huge number of features of the road, tire, and suspension; however, it can be wellapproximated as follows:

$$
F_{x}=F_{z} \mu\left(\lambda, \beta_{t} ; \theta_{r}\right) \text {. }
$$

In (2) $F_{z}$ is the vertical force at the tire-road contact point; $\lambda$ is the longitudinal slip; $\beta_{t}$ is the side-slip angle of the wheel; $\theta_{r}$ is a set of parameters which characterize the shape of $\mu(\cdot)$.

Expression (2) can be further elaborated. The vertical load can be simply described as $F_{z}=m g$, while the longitudinal slip is given by

$\lambda=\frac{v-\omega r}{v}=1-r \frac{\omega}{v}$.
In the rest of the paper it is assumed that the braking maneuver is performed along a straight line. Accordingly, the dependence of $F_{x}$ on $\beta_{t}$ will be omitted:

$F_{X}=m g \mu\left(\lambda ; \theta_{r}\right)=m g \mu\left(\frac{v-\omega r}{v} ; \theta_{r}\right)$.

A simple and widely-used model for $\mu(\cdot)$ is:

$\mu\left(\lambda ; \theta_{r}\right)=\theta_{r 1}\left(1-\exp \left(-\lambda \theta_{r 2}\right)\right)-\lambda \theta_{r 3}$.

By changing the values of these parameters, many different road conditions can be modeled (Fig.2).

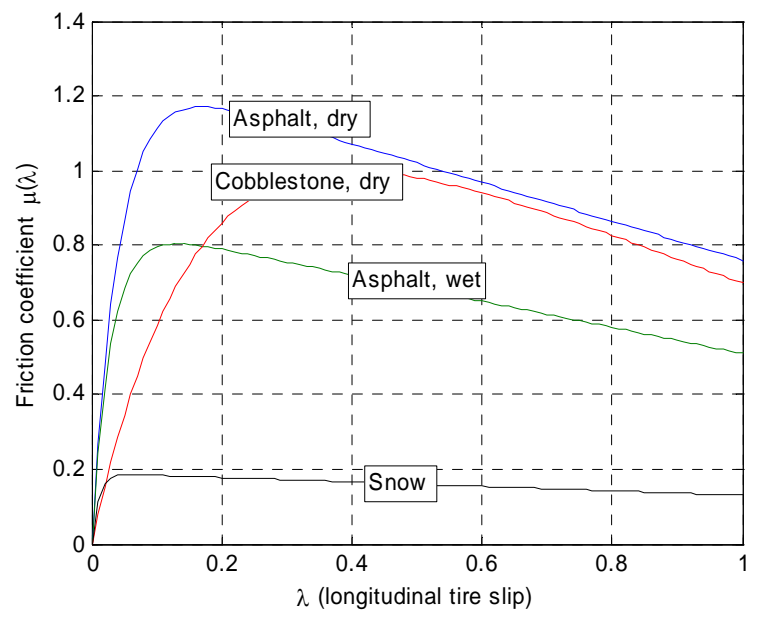

Fig.2. Shapes of $\mu(\lambda)$ in different road conditions.

By plugging (4) into (1), we finally obtain the following expression of the quarter-car model:

$\left\{\begin{array}{l}J \dot{\omega}=r m g \mu\left(\frac{v-\omega r}{v}\right)-T_{b} \\ m \dot{v}=-m g \mu\left(\frac{v-\omega r}{v}\right)\end{array}\right.$

As already remarked, in (6a) the state variables are $\omega$ and $v$. Since $\omega, v$ and $\lambda$ are linked by the algebraic relationship (3), it is possible to replace the state variable $\omega$ with $\lambda$. This can be obtained by plugging the following two relationships

$\dot{\lambda}=-\frac{r}{v} \dot{\omega}+\frac{r \omega}{v^{2}} \dot{v}, \quad \omega=\frac{v}{r}(1-\lambda)$

into the first equation of (6a), so obtaining:

$\left\{\begin{array}{l}\dot{\lambda}=-\frac{1}{v}\left(\frac{(1-\lambda)}{m}+\frac{r^{2}}{J}\right) m g \mu(\lambda)+\frac{r}{v J} T_{b} \\ m \dot{v}=-m g \mu(\lambda)\end{array}\right.$

\section{ANALYSIS OF THE LINEARIZED MODEL}

The first step of our analysis is the computation and discussion of the equilibrium points for (6).

First notice that, by setting in (6) $\dot{v}=0$ and $\dot{\omega}=0$, the corresponding equilibrium is given by $\mu=0$ and $T_{b}=0$. This equilibrium condition is meaningless for the design of a braking regulation loop. The equilibrium points we are interested in are characterized by $\dot{\lambda}=0$ and $\eta=\bar{\eta}$. Starting from 
these conditions, it is possible to find the set of admissible equilibrium points. Consider the expression (3) of $\lambda$. By setting $\dot{\lambda}=0$ we obtain $\dot{\omega}=\dot{v} \omega / v$. By replacing $\omega=v / r(1-\lambda)$, $m \dot{v}=-m g \mu(\lambda)$, and $\eta=-\dot{\omega} r / g$ into this expression, we obtain the analytic expression of the set of admissible equilibrium points in the $(\lambda, \eta)$ plane:

$\bar{\eta}=(1-\bar{\lambda}) \mu(\bar{\lambda})$.

In Fig.3 the equilibrium manifold (7) is displayed.

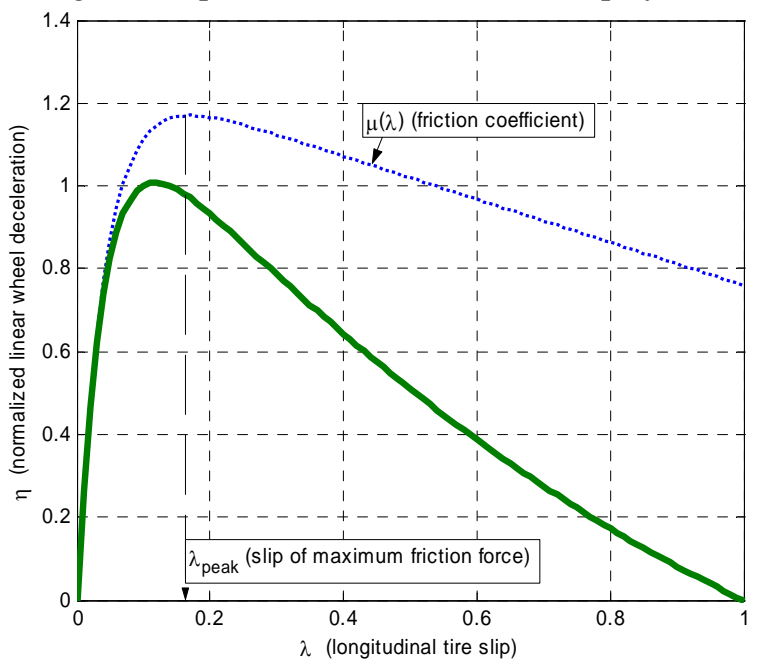

Fig.3. Equilibrium manifold $\bar{\eta}(\bar{\lambda})$ (dry asphalt).

In order to linearize the model (6) around an equilibrium point (Guardabassi and Savaresi, 2001), it is assumed that the longitudinal dynamics of the vehicle are much slower than the rotational dynamics of the wheel. Henceforth, $v$ is considered as a slowly-varying parameter. Under this assumption the second equation of (6) is neglected, and the model reduces to a simple $1^{\text {st }}$ order model of the wheel dynamics. Consider now the variables $\delta T_{b}=T_{b}-\bar{T}_{b}$, $\delta \lambda=\lambda-\bar{\lambda}, \quad \delta \dot{\omega}=\dot{\omega}-\overline{\dot{\omega}}, \quad \delta \eta=\eta-\bar{\eta}$, defined around an equilibrium point. Consider also the following definition:

$\mu_{1}(\bar{\lambda})=\partial \mu(\lambda) /\left.\partial \lambda\right|_{\lambda=\bar{\lambda}}$.

Using the above variables and the definition of $\mu_{1}(\bar{\lambda})$, the first equation of (6b) can be linearized; the following linear dynamic equation is obtained:

$\delta \dot{\lambda}=\left[\frac{1}{v}\left(\frac{m g \mu(\bar{\lambda})}{m}-m g \mu_{1}(\bar{\lambda})\left(\frac{(1-\lambda)}{m}+\frac{r^{2}}{J}\right)\right)\right] \delta \lambda+\left[\frac{r}{v J}\right] \delta T_{b}$.

The transfer function $G_{\lambda}(s)$ from $\delta T_{b}$ to $\delta \lambda$ is given by:

$$
G_{\lambda}(s)=\frac{\left[\frac{r}{v J}\right]}{s+\left[\frac{1}{v}\left(g \mu_{1}(\bar{\lambda})\left((1-\bar{\lambda})+\frac{m r^{2}}{J}\right)-g \mu(\bar{\lambda})\right]\right.} \cdot
$$

The transfer function $G_{\eta}(s)$ from $\delta T_{b}$ to $\delta \eta$ can be obtained by linearizing the first equation of (6a):

$$
G_{\eta}(s)=\frac{\frac{r}{J g}\left(s+\left[\frac{g}{v}\left(\mu_{1}(\bar{\lambda})(1-\bar{\lambda})-\mu(\bar{\lambda})\right)\right]\right)}{s+\left[\frac{1}{v}\left(g \mu_{1}(\bar{\lambda})\left((1-\bar{\lambda})+\frac{m r^{2}}{J}\right)-g \mu(\bar{\lambda})\right]\right]} .
$$

The analysis of $G_{\lambda}(s)$ and $G_{\eta}(s)$ explains the behavior of the open-loop braking dynamics.

The pole of $G_{\lambda}(s)$ and $G_{\eta}(s)$ is given by:

$$
s_{\text {pole }}=-\frac{1}{v}\left(\mu_{1}(\bar{\lambda})\left((1-\bar{\lambda})+\frac{m r^{2}}{J}\right)-\mu(\bar{\lambda})\right) g \text {; }
$$

the zero of $G_{\eta}(s)$ is given by:

$$
s_{\text {zero }}=-\frac{1}{v}\left(\mu_{1}(\bar{\lambda})(1-\bar{\lambda})-\mu(\bar{\lambda})\right) g \text {. }
$$

By studying the pole and the zero locations, the stability and minimum-phase properties of the system around a steady-state condition can be analyzed.

The stability condition for $G_{\lambda}(s)$ and $G_{\eta}(s)$ is:

$$
\mu_{1}(\bar{\lambda})\left((1-\bar{\lambda})+\frac{m r^{2}}{J}\right)>\mu(\bar{\lambda}) \text {. }
$$

However, note that, since $(1-\bar{\lambda})<<m r^{2} / J$ and $J \mu(\bar{\lambda}) /\left(m r^{2}\right) \approx 0$, it can be reduced to $\mu_{1}(\bar{\lambda})>0$. This means that $G_{\lambda}(s)$ and $G_{\eta}(s)$ are unstable if the equilibrium point $\bar{\lambda}$ is beyond the peak of $\mu(\lambda)$.

The minimum-phase condition for $G_{\eta}(s)$ is:

$\mu_{1}(\bar{\lambda})>\frac{\mu(\bar{\lambda})}{(1-\bar{\lambda})}$.

This analysis has shown that the equilibrium condition $\bar{\lambda}$ strongly affects the stability and minimum-phase properties of $G_{\lambda}(s)$ and $G_{\eta}(s)$. It also shows that the open-loop wheel dynamics can be unstable. This instability can hardly be handled by non-professional drivers, and represents the main motivation for the design of electronic Anti-lock Braking Systems.

\section{CONTROL STRATEGIES}

The general structure of the proposed Mixed SlipDeceleration (MSD) control scheme is outlined in Fig.4. The basic idea is to define an output controlled variable $\varepsilon$, which is the convex combination of the two measured variables $\eta$ and $\lambda$, namely:

$\varepsilon=\alpha \lambda+(1-\alpha) \eta, \alpha \in[0,1]$,

and to regulate this variable to a set-point constant value $\bar{\varepsilon}$. Also $\bar{\varepsilon}$ can be interpreted as $\bar{\varepsilon}=\alpha \bar{\lambda}+(1-\alpha) \bar{\eta}$.

The control variable $\delta T_{b}$ is assumed to be driven by the regulation error through a proportional controller, namely $\delta T_{b}=K \delta \varepsilon$ (where $\delta \varepsilon=\varepsilon-\bar{\varepsilon}$ ). Note that the choice of a proportional controller has been made to keep the overall control scheme as simple as 
possible, in order to easily gain some insight in the behavior of the control system. The proportional controller can be upgraded with more sophisticated SISO controllers.

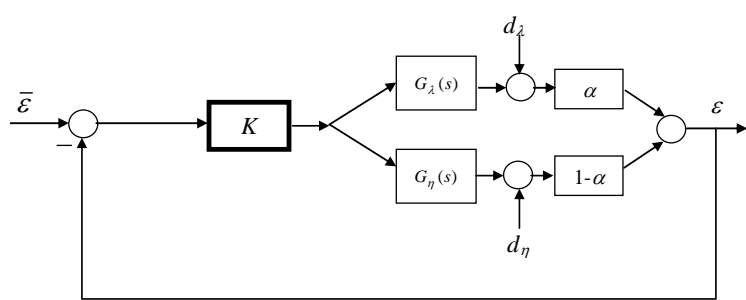

Fig.4. General scheme of the MSD controller.

The MSD controller displayed in Fig.4 has the peculiar feature of embedding the slip-controller ( $\alpha=1)$ and the deceleration-controller $(\alpha=0)$.

\section{1. $\alpha=1$ (Slip control)}

By setting $\alpha=1$ in (10), the controlled variable is the wheel slip $\lambda$. Note that (see Fig.2), whatever $\bar{\lambda}$ is $(0 \leq \bar{\lambda} \leq 1)$, this regulation scheme guarantees the uniqueness of the steady-state solution. In order to analyze the dynamic properties of the slip control system, it is useful to compute the open-loop transfer function:

$$
L_{\lambda}(s)=\frac{K\left[\frac{r}{v J}\right]}{s+\left[\frac{1}{v}\left(g \mu_{1}(\bar{\lambda})\left((1-\bar{\lambda})+\frac{m r^{2}}{J}\right)-g \mu(\bar{\lambda})\right)\right]} .
$$

The following stability condition can be worked out.

\section{Stability condition for slip-control}

$$
\frac{K r}{J}>g \mu(\bar{\lambda})-g \mu_{1}(\bar{\lambda})\left((1-\bar{\lambda})+\frac{m r^{2}}{J}\right) \text {. }
$$

The analysis of (11) reveals that it is always possible to find a value $\bar{K}$ such that, for $K>\bar{K}$, the closedloop system is stable in every condition (namely for every value of $\bar{\lambda}$ and for every road condition).

This analysis explains why slip-control is considered a very attractive control approach:

- Given a set-point $\bar{\lambda}$, it guarantees the uniqueness of the steady-state solution.

- The choice of $\bar{\lambda}$ is not critical; as a matter of fact, it is easy to find a value of $\bar{\lambda}$ (e.g. $\bar{\lambda}=0.15$ ) which provides very good results for every road condition.

- With a fixed-controller structure, the asymptotic stability of the closed-loop is guaranteed for every value of $\bar{\lambda}$ and for every road condition.

The major drawback of slip-control is that the measurement of $\lambda$ is difficult and unreliable. As a matter of fact the wheel slip computation requires the measurement of the vehicle longitudinal speed, and the vehicle speed can only be estimated by indirect measurements.

\section{2. $\alpha=0$ (Deceleration control)}

By setting $\alpha=0$ in (10), the controlled variable is the normalized linear wheel deceleration $\eta$. From Fig.2, it is clear the first major drawback of deceleration control: the selection of the set-point $\bar{\eta}$ is very critical, and it is impossible to find an unique value of $\bar{\eta}$ which gives a good compromise for every road condition. As already remarked, note that (if any) the system always has two equilibrium points.

In order to analyze the dynamic properties of the deceleration control system, it is useful to compute the open-loop transfer function:

$$
L_{\eta}(s)=\frac{K \frac{r}{J g}\left(s+\left[\frac{g}{v}\left(\mu_{1}(\bar{\lambda})(1-\bar{\lambda})-\mu(\bar{\lambda})\right)\right]\right)}{s+\left[\frac{1}{v}\left(g \mu_{1}(\bar{\lambda})\left((1-\bar{\lambda})+\frac{m r^{2}}{J}\right)-g \mu(\bar{\lambda})\right]\right]} .
$$

The following stability condition can be worked out:

$$
\begin{aligned}
& \text { Stability condition for deceleration-control } \\
& g \mu_{1}(\bar{\lambda}) \frac{m r^{2}}{J}+K \frac{r}{J}\left(\mu_{1}(\bar{\lambda})(1-\bar{\lambda})-\mu(\bar{\lambda})\right)>0 .
\end{aligned}
$$

Note that it is not possible to find a fixed value of $K$ which provides stability for every value of $\bar{\lambda}$ and for every road condition: if $K>0$, the system can be made asymptotically stable before the friction-curve peak, but it becomes unstable beyond the peak. Henceforth, one of the two equilibrium points is always unstable.

This analysis explains the main limits of deceleration-control:

- The choice of $\bar{\eta}$ is very critical; henceforth, it must be adapted on-line, by means of a detection algorithm of the road conditions.

- With a fixed-controller structure the asymptotic stability of the closed-loop system is not guaranteed.

Due to these major drawbacks, deceleration-control has never been implemented as a classical regulation loop: complex rule-based algorithms based on a set of adjustable thresholds on $\eta$ and its derivative have been traditionally implemented. These approaches provide acceptable results if anti-locking is the only objective, but can hardly be used for more sophisticated Electronic Stability Control systems.

On the other hand, the wheel deceleration can be measured in a very reliable and straightforward manner: it is a low-cost measurement, the noise $d_{\eta}$ affecting the measure of $\eta$ is almost stationary, and the variance of this noise can be easily managed by properly choosing the precision of the wheel encoder.

\section{3. $0<\alpha<1$ (Mixed Slip-Deceleration control)}

In this case, the controlled variable is $\varepsilon=\alpha \lambda+(1-\alpha) \eta$, and the set-point is $\bar{\varepsilon}=\alpha \bar{\lambda}+(1-\alpha) \bar{\eta}$. The graphical interpretation of this control strategy in the $(\lambda, \eta)$ domain is displayed in Fig.5. Clearly, by carefully choosing $\alpha$ and $\bar{\varepsilon}$, it 
is possible to guarantee the existence and uniqueness of the steady-state condition; moreover, it is easy to find a fixed value of $\alpha$ and $\bar{\varepsilon}$ such that, for every road condition, the equilibrium point provides good performance.

In this case, the open-loop transfer function is given by:

$$
L_{\varepsilon}(s)=K \frac{\alpha\left[\frac{r}{v J}\right]+(1-\alpha) \frac{r}{J g}\left(s+\left[\frac{g}{v}\left(\mu_{1}(\bar{\lambda})(1-\bar{\lambda})-\mu(\bar{\lambda})\right)\right]\right)}{s+\left[\frac{1}{v}\left(g \mu_{1}(\bar{\lambda})\left((1-\bar{\lambda})+\frac{m r^{2}}{J}\right)-g \mu(\bar{\lambda})\right]\right]}
$$

The following stability condition can be worked out.

\section{Stability condition for MSD-control}

If the parameter $\alpha$ is chosen such that:

$\alpha_{\min }<\alpha \leq 1$,

where

$$
\alpha_{\min }=\max _{\substack{\bar{\lambda} \\ \theta_{r}}}\left\{\frac{\left[\mu\left(\bar{\lambda} ; \theta_{r}\right)-\mu_{1}\left(\bar{\lambda} ; \theta_{r}\right)(1-\bar{\lambda})\right]}{1+\left[\mu\left(\bar{\lambda} ; \theta_{r}\right)-\mu_{1}\left(\bar{\lambda} ; \theta_{r}\right)(1-\bar{\lambda})\right]}\right\},
$$

it is always possible to find a value $\bar{K}$ such that, for $K>\bar{K}$, the closed-loop system is stable in every condition (for every value of $\bar{\lambda}$ and for every road condition).

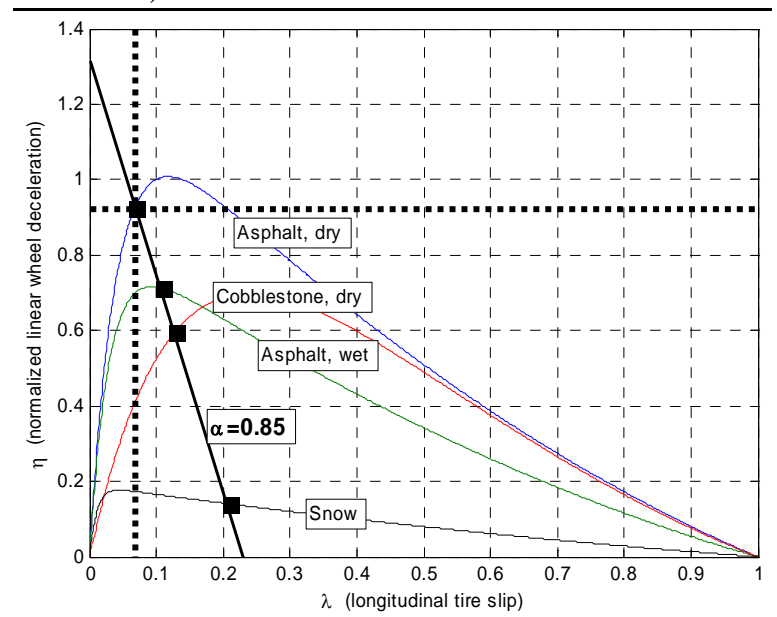

Fig.5. Graphical interpretation of MSD control.

Given the previous stability results for slip-control and deceleration-control, the above condition (14) could be somehow expected: we have seen that for $\alpha=1$ stability can be always guaranteed, whereas for $\alpha=0$ it is impossible to find a unique globallystable proportional controller. For continuity, these two extremal conditions must be separated by a "threshold" (or lower-bound) on $\alpha$, located between 0 and 1. Expression (14) provides the analytic expression of this lower bound $\alpha_{\min }$.

Note that the lower bound is the worst case with respect to the equilibrium point $(\bar{\lambda})$ and the road conditions $\left(\theta_{r}\right)$. Also note that the worst condition occurs when $\mu\left(\bar{\lambda} ; \theta_{r}\right)$ is big and $\mu_{1}\left(\bar{\lambda} ; \theta_{r}\right)$ is negative. A rough estimation for $\alpha_{\min }$ is $\alpha_{\min } \approx 0.6$. At the end of this analysis, one can conclude that, if $\alpha_{\text {min }}<\alpha<1$, the MSD control strategy essentially has the same appealing features of slip-control. However, at a first sight, MSD-control also seems to share the major drawback of slip-control (sensitivity to poor slip measurements). This issue will be discussed in the following subsection.

\subsection{Disturbance analysis}

Consider the general MSD in Fig.4. It is easy to see that the disturbance $d_{\varepsilon}$ affecting the closed-loop controlled variable is given by:

$$
D_{\varepsilon}(s)=\alpha \frac{1}{1+L_{\varepsilon}(s ; \alpha)} D_{\lambda}(s)+(1-\alpha) \frac{1}{1+L_{\varepsilon}(s ; \alpha)} D_{\eta}(s)
$$

where $L_{\varepsilon}(s)$ is the open-loop transfer function of the MSD control scheme, given by (13), and $D_{\varepsilon}(s), D_{\lambda}(s), D_{\eta}(s)$ are the Laplace transform of the signals $d_{\varepsilon}, d_{\lambda}, d_{\eta}$, respectively.

Assume now that $d_{\lambda}$ and $d_{\eta}$ are zero-mean, uncorrelated, band-limited white noises (they have no spectral content beyond $\bar{\Omega}$ ). For the sake of simplicity, assume also that the variances of $d_{\lambda}$ and $d_{\eta}$ are the same, namely $\operatorname{var}\left[d_{\lambda}\right]=\operatorname{var}\left\lfloor d_{\eta}\right\rfloor=\Psi$. Under these assumptions, the variance of $d_{\varepsilon}$ is:

$$
\operatorname{var}\left[d_{\varepsilon}(\alpha)\right]=\Psi\left(\alpha^{2}+(1-\alpha)^{2}\right) \int_{w=0}^{\bar{\Omega}}\left|\frac{1}{1+L_{\varepsilon}(j w, \alpha)}\right|^{2} d w
$$

In order to understand if the MSD approach outperforms the slip-control approach, we must prove that

$$
\operatorname{var}\left[d_{\varepsilon}(\alpha)\right]<\operatorname{var}\left[d_{\varepsilon}(1)\right], \quad \alpha_{\text {min }}<\alpha<1 .
$$

Note that (15) is the product of two terms, both depending on $\alpha$. The analysis of the first term $\left(\alpha^{2}+(1-\alpha)^{2}\right)$ is very simple. This function is maximum for $\alpha=1$ (slip control) and $\alpha=0$ (deceleration control). MSD control (namely $0<\alpha<1$ ) hence provides a noise attenuation which - considering this term only - can halve the noise variance on the controlled variable. In particular, at $\alpha=\alpha_{\text {min }}=0.6$, the attenuation factor given by this term is about 0.52 . The analysis of second factor of (15) is more complicated since its explicit expression cannot be analytically computed. Note that this term is the integral (over the frequency interval $[0, \bar{\Omega}]$ ) of the squared magnitude of the frequency response of the closed-loop sensitivity function; a clear indication on the effect of $\alpha$ on this term hence can be drawn by the analysis of the sensitivity function. The sensitivity transfer function can be condensed in four basic features: Zero, Pole, Low-Frequency (LF) gain and High-Frequency (HF) gain.

Zero: $-\left[\frac{1}{v}\left(g \mu_{1}(\bar{\lambda})\left((1-\bar{\lambda})+\frac{m r^{2}}{J}\right)-g \mu(\bar{\lambda})\right)\right]$; 
Pole: $\frac{\left[\frac{1}{v}\left(g \mu_{1}(\bar{\lambda})\left((1-\bar{\lambda})+\frac{m r^{2}}{J}\right)-g \mu(\bar{\lambda})+K(1-\alpha) \frac{r}{J}\left(\mu_{1}(\bar{\lambda})(1-\bar{\lambda})-\mu(\bar{\lambda})\right)+\frac{K o r}{J}\right)\right]}{\left[1+K(1-\alpha) \frac{r}{J g}\right]} ;$

LF gain:

$$
\frac{g \mu_{1}(\bar{\lambda})\left((1-\bar{\lambda})+\frac{m r^{2}}{J}\right)-g \mu_{(\bar{\lambda})}}{g \mu_{1}(\bar{\lambda})\left((1-\bar{\lambda})+\frac{m r^{2}}{J}\right)-g \mu_{(}(\bar{\lambda})+K(1-\alpha) \frac{r}{J}\left(\mu_{1}(\bar{\lambda})(1-\bar{\lambda})-\mu(\bar{\lambda})\right)+\frac{K o r}{J}}
$$

HF gain: $\frac{1}{\left[1+K(1-\alpha) \frac{r}{J g}\right]}$.

From the analysis of these four features with respect to $\alpha$ (assuming high values of $K$ ), the following remarks can be done:

- The Zero is fixed, and does not depend on $\alpha$.

- The Pole shifts backwards as $\alpha$ decreases;

- The LF gain is only weakly affected by $\alpha$;

- The HF-gain strongly decreases as $\alpha$ decreases.

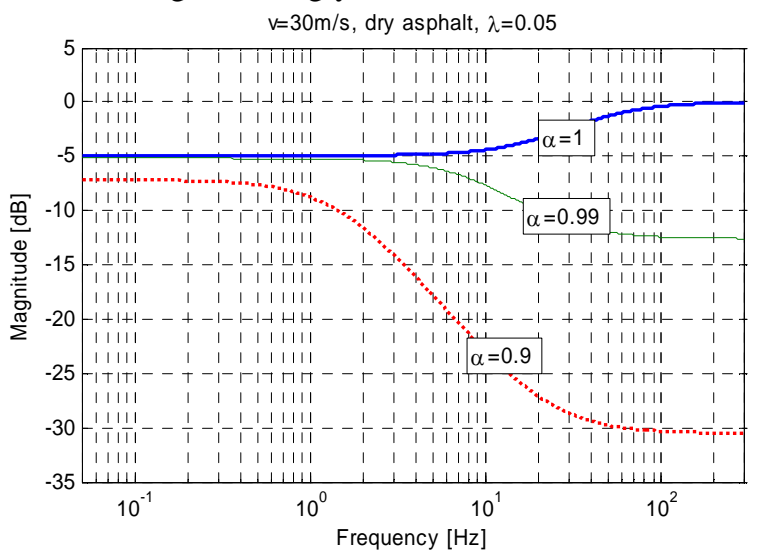

Fig.6. Bode-plots of (26) when $\bar{\lambda}=0.05$.

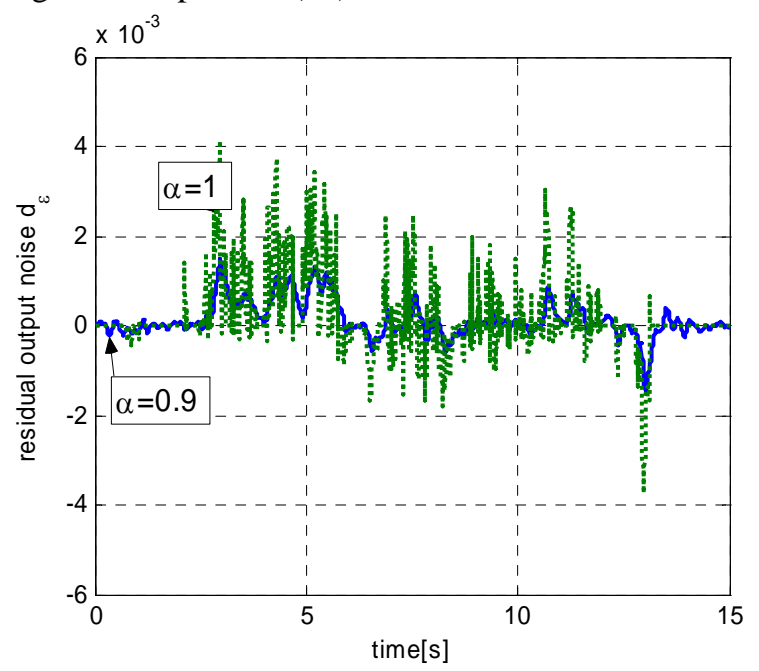

Fig.7. Closed-loop noise on the controlled variable.

All these characteristics can be easily understood by means of Fig.6, where the magnitude of the frequency response of the sensitivity function is displayed for three different values of $\alpha$. All the characteristics outlined above are clearly visible in this picture. Similar plots can be obtained for different road conditions and different longitudinal vehicle speeds.
Finally, this noise-attenuation effect can be appreciated by the simulation results displayed in Fig.7, where $d_{\varepsilon}$ is displayed, in the case of $\alpha=1$ and $\alpha=0.9$ (dry asphalt condition, $\bar{\lambda}=0.05$, $v=30 \mathrm{~m} / \mathrm{s}, K=10000)$. It is clear the advantage of using a MSD control strategy. This result has been obtained by using the measurement errors on $\lambda$ and $\eta$ registered during an experiment on a real vehicle.

\section{ACKNOWLEDGMENTS}

This work has been supported by BREMBO S.p.A. and MIUR project "New methods for Identification and Adaptive Control for Industrial Systems". Thanks are due to Michele Besio and Valerio Zanella.

\section{REFERENCES}

Drakunov S., U. Ozguner, P. Dix, B. Ashrafi (1995). "ABS Control Using Optimum Search via Sliding Modes”. IEEE Transactions on Control system Technologies, Vol. 3, No.1, pp.79-85.

Gissinger G.L., C.Menare, A.Constans (2003). “A mechatronic conception of a new intelligent braking system”. Control Engineering Practice, Vol.11, pp.163 -170.

Guardabassi G., S.M. Savaresi (2001). Approximate Linearization via Feedback - an Overview. Automatica, survey paper, vol.27, pp.1-15.

Gustafsson F. (1997). "Slip-based Tire-Road Friction Estimation”. Automatica, Vol.33, pp. 1087-1099.

Johansen T.A., Petersen I., Kalkkuhl J., Lüdemann J. (2003). "Gain-Scheduled Wheel Slip Control in Automotive Brake Systems”. IEEE Trans. on Control Systems Technology, Vol. 11, No. 6, pp.799-811.

Kiencke U., Nielsen L. (2000). “Automotive Control Systems for Engine, Driveline, and Vehicle”. Springer Verlag.

Layne J. R., Passino K. M., Yurkovich S. (1993), "Fuzzy Learning Control for Antiskid Braking Systems," IEEE Transactions on Control Systems Technology, Vol. 1, No. 2, pp. 122-129.

Ono E., Asano K., Sugai M., Ito S., Yamamoto M., Sawada M., Yasui Y. (2003). "Estimation of automotive tire force characteristics using wheel velocity”. Control Engineering Practice, Vol.11, pp.1361-1370.

Solyom S., Rantzer A., Lüdemann J. (2004). "Synthesis of a Model-based Tire Slip Controller." Journal of Vehicle System Dynamics, Vol. 41, No 6, pp. 477-511.

Wellstead P.E., Petit N. B., (1997). “Analysis and Redesign of an Antilock brake system controller”. In Proc. of the Inst. of Elect. Eng., Vol.144, pp.413-426.

Yi J., Alvarez L., Horowitz R, (2002). “Adaptive Emergency Braking Control With Underestimation of Friction Coefficient”. IEEE Transactions on Control System Technology, Vol. 10, No. 3, pp. 381-392. 